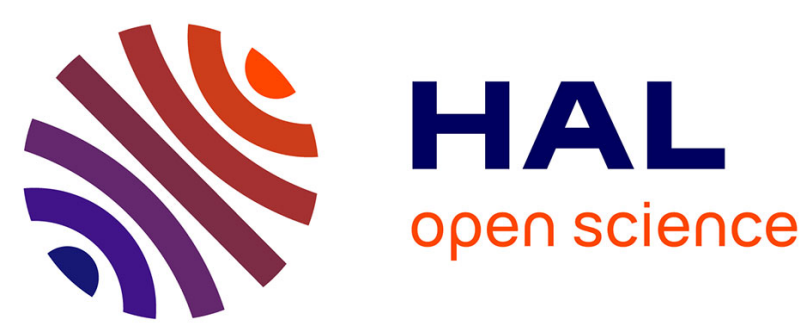

\title{
Les équipements de D.F.C.I
}

Roland Croisé

\section{To cite this version:}

Roland Croisé. Les équipements de D.F.C.I. Revue forestière française, 1975, 27 (S), pp.298-299. $10.4267 / 2042 / 20983$. hal-03396310

\section{HAL Id: hal-03396310 https://hal.science/hal-03396310}

Submitted on 22 Oct 2021

HAL is a multi-disciplinary open access archive for the deposit and dissemination of scientific research documents, whether they are published or not. The documents may come from teaching and research institutions in France or abroad, or from public or private research centers.
L'archive ouverte pluridisciplinaire HAL, est destinée au dépôt et à la diffusion de documents scientifiques de niveau recherche, publiés ou non, émanant des établissements d'enseignement et de recherche français ou étrangers, des laboratoires publics ou privés. 


\section{Les équipements de D.F.C.I.}

Comme toute action collective destinée à combattre un fléau ou à en réparer les effets, la défense contre les incendies de forêt ne se conçoit pas sans un plan définissant des moyens, avec toutes les conditions de leur intervention et de leur mise en œuvre. Ces moyens sont évoqués et examinés dans le détail dans les différentes études de ce numéro spécial, mais il n'est sans doute pas superflu de souligner ce qui cause leur interindépendance et entraîne leur nécessaire coordination.

Suivant les circonstances, un feu - presque toujours insignifiant à sa naissance — peut n'être qu'un accident aux conséquences minimes ou bien se transformer en un sinistre grave. Certains facteurs de son développement échappent entièrement au pouvoir de I'homme, comme les conditions météorologiques ou les caractéristiques du terrain. L'homme peut influer sur certains autres facteurs, par exemple en modifiant peu à peu la composition floristique et surtout la structure des peuplements en vue de ralentir la propagation du feu. II est maître enfin d'une troisième catégorie de facteurs : il peut tenter de limiter l'extension d'un feu futur par l'ouverture préalable de bandes dénudées : les pare-feu; il peut aussi intervenir directement contre le feu lui-même, avec des moyens plus ou moins importants : c'est la lutte active, qui nécessite des équipements constituant une mise en état de défense préalable et permanente de la forêt.

Lorsqu'un incendie est déclaré, les moyens de lutte se déploient en fonction, d'une part des conditions de l'incendie lui-même : point de départ, intensité, vitesse de propagation, et d'autre part des conditions et lignes caractéristiques du terrain, naturelles (talwegs, crêtes) ou artificielles (pare-feu, chemins).

L'un des facteurs déterminants de l'efficacité de la lutte active proprement dite — presque plus important que la somme des moyens mis en œuvre - est la rapidité avec laquelle la lutte est engagée. Les équipements sont donc conçus avec cette préoccupation dominante : permettre aux moyens de parvenir à leur lieu d'emploi dans le plus bref délai possible à partir de la naissance d'un feu.

La défense forestière contre l'incendie s'inspire de ces idées de base et s'articule généralement autour d'un schéma devenu classique, et même évident, dans plusieurs régions où son efficacité a été bien prouvée :

- quadrillage du terrain par des coupures, les pare-feu;

- moyens assurant une détection la plus rapide possible d'un foyer d'incendie grâce à une surveillance terrestre (permanente en période dangereuse), complétée éventuellement par une surveillance aérienne ;

- possibilités de mise sur pied immédiate d'unités de lutte, avec personnel et matériel (en état d'alerte permanente en période dangereuse);

- possibilités d'acheminement rapide de ces moyens sur les zones où la lutte peut être engagée avec la meilleure efficacité (équipement routier);

— possibilités d'approvisionnement facile et rapide en eau.

Les moyens aériens de lutte, qui viennent en complément de ces plans, sont de nature à renforcer sensiblement l'efficacité des moyens d'intervention; en toute situation, ils permettent une action rapide et concentrée en n'importe quel point, ce qui est précieux pour les zones d'approche difficile; là où l'infrastructure - notamment l'infrastructure routière - est insuffisante, ils peuvent exercer l'action prépondérante ou même constituer la seule forme d'action possible.

* D.F.C.I. : Défense des forêts contre l'incendie. 
Pour l'application du plan de défense contre l'incendie, il faut prévoir toutes les situations qui peuvent se présenter, envisager les solutions à leur apporter et en déduire un programme d'équipement adapté à la situation. Si minutieuses que soient ces études de prévisions et simulations, elles ne peuvent cependant que donner des indications sur les caractères probables des incendies futurs, les zones de départ les plus fréquentes, les directions vraisemblables de propagation, c'est-à-dire que les plans opératoires basés sur ces études ne peuvent pas ne pas avoir un caractère un peu aléatoire. II n'en est que plus évident qu'une bonne efficacité ne peut être obtenue qu'au prix d'une coordination poussée de tous les éléments à mettre en œuvre, et en particulier des éléments de l'infrastructure : postes de guet, pare-feu, routes, points d'eau, aires d'atterrissage pour hélicoptères.

II est clair aussi qu'on ne peut concevoir de plan d'équipement dans l'abstrait, et qu'il ne peut exister un plan uniforme, applicable à tous les terrains et à toutes les situations.

Dans le secteur où le schéma général a été mis au point, la forêt landaise, le relief est faible ou quasi inexistant et le sol, sableux, facile à travailler : il a été possible d'y asseoir un quadrillage régulier et géométrique, compartimentant un terrain facile à surveiller en aires dont le repérage et l'accès sont aisés. Cela n'est plus possible en forêt montagneuse, et particulièrement en forêt méditerranéenne où beaucoup de conditions défavorables sont rassemblées : relief découpé, avec des vallons profonds et des angles morts pour l'observation terrestre ; sécheresse estivale très marquée et vents très violents, aggravant I'inflammabilité d'une végétation xérophile; réseau routier peu développé et d'un coût de construction élevé ; rareté et insuffisance quantitative des points d'eau; enfin, implantation humaine irrégulièrement répartie sur le terrain et morcellement accentué d'une propriété boisée de faible valeur, ce qui a paralysé le développement de travaux d'équipement d'intérêt général; dans certaines zones particulièrement difficiles, seules les interventions aériennes peuvent être actuellement envisagées.

C'est dans ces forêts que les plans d'équipements de défense contre les incendies de forêt doivent être le plus minutieusement élaborés, et toujours dans le cadre d'études pluridisciplinaires. Ils ne constituent qu'une partie des actions de grande envergure et de longue durée qui seront à mener, au prix d'efforts importants et coûteux, de la part de tous les organismes et services responsables, concertés à cette occasion. Les valeurs à préserver sont à la hauteur des sacrifices à consentir et leur caractère irremplaçable est, heureusement, de mieux en mieux compris.

Roland CROISÉ

Ingénieur en chef du G.R.E.F.

Chef de la division

"Équipement et exploitation des forêts "

Groupement de Nogent-sur-Vernisson du

CENTRE TECHNIQUE DU GÉNIE RURAL, DES EAUX ET DES FORETS

Domaine des Barres

45290 NOGENT-SUR-VERNISSON 